Research Article

\title{
Some Results on Generalized Pata-Suzuki Type Contractive Mappings
}

\author{
Merve Aktay $(\mathbb{D}$ and Murat Özdemir \\ Department of Mathematics, Faculty of Science, Atatürk University, Erzurum 25240, Turkey \\ Correspondence should be addressed to Merve Aktay; merve.ozkan@atauni.edu.tr
}

Received 14 April 2021; Accepted 27 May 2021; Published 9 June 2021

Academic Editor: Zoran Mitrovic

Copyright ( $) 2021$ Merve Aktay and Murat Özdemir. This is an open access article distributed under the Creative Commons Attribution License, which permits unrestricted use, distribution, and reproduction in any medium, provided the original work is properly cited.

In this work, we establish new fixed point theorems for generalized Pata-Suzuki type contraction via $\alpha$-admissible mapping in metric spaces and to prove some fixed point results for such mappings. Moreover, we give an example to illustrate our main result. Consequently, the results presented in this paper generalize and improve the corresponding results of the literature.

\section{Introduction and Preliminaries}

The Banach contraction principle was introduced by Banach [1] which is one of the earlier and the most important result in fixed point theory. Because of its importance, over the years, many authors extended and generalized the Banach contraction principle in many directions.

The concept of almost contraction was given by Berinde and Pacurar [2]. Also this concept was compared with other contractions and proved some fixed point theorems by Berinde ([2-4]).

$C$-condition was introduced by Suzuki ([5-7]). Suzuki [8] proved generalized versions of Edelstein theorem [9] for compact metric spaces.

Firstly, Samet et al. [10] introduced an interesting contraction which is called $\alpha-\varrho-$ contractive and gave $\alpha$-admissible mappings and investigated the existence and uniqueness of such mappings in the setting of complete metric spaces. Later, in addition to Samet et al. [10], some different fixed point theorems were introduced by Karapinar and Samet [11] and Babu et al. [12].

Recently, one of the famous generalizations of the Banach contraction principle for the existence and uniqueness of fixed points for self mappings on metric spaces is the theorem by Pata [13]. Since Pata's fixed point theorem, some authors have studied this theorem in several ways (see [14-18]).

Using the ideas of Pata [13], we give a generalization of Pata-type contractions. The purpose of this paper is to introduce almost $(\alpha, \varrho)$-Pata-Suzuki type contractive mapping and to prove some fixed point results for such mappings with admissibility condition. These results are generalizations of various results in the literature.

Now, we give some definitions and fundamental results.

Let $(E, \rho)$ be a metric space; a point $u \in E$ is said to be a fixed point of $h: E \longrightarrow E$ if $u=h u$. The set of all fixed points of $f$ is denoted by $\operatorname{Fix}(h)$.

Berinde and Pacurar [2] introduced the concept of almost contraction as a generalization of Banach contraction principle.

Definition 1 (see [2]). Let $(E, \rho)$ be a metric space and $h: E \longrightarrow E$ be a mapping which is called an almost contraction if there exists a constant $\vartheta \in(0,1)$ and $L \geq 0$ such that

$$
\rho(h t, h s) \leq \vartheta(t, s)+L \rho(s, h t),
$$

for all $t, s \in E$.

In 2011, Pata [13] proved the following result. 
Theorem 1 (see [13]). Let $(E, \rho)$ be a complete metric space and $\Psi$ denote the class of all increasing function $\psi:[0,1] \longrightarrow[0, \infty)$, which vanishes with continuity at zero. For an arbitrary $t_{0} \in E$, we denote $\|t\|=\rho\left(t, t_{0}\right), \forall t \in E$. Let $\Lambda \geq 0, \xi \geq 1$, and $\beta \in[0, \xi]$ be fixed constants and $\psi \in \Psi$ and $f: E \longrightarrow E$ be a function. If for all $t, s \in E$, the following inequality is satisfied:

$$
\rho(h t, h s) \leq(1-\varepsilon) \rho(t, s)+\Lambda \varepsilon^{\xi} \psi(\varepsilon)[1+\|t\|+\|s\|]^{\beta},
$$

for all $\varepsilon \in[0,1]$, and then $h$ has a unique fixed point $u=h u$.

Suzuki ([5-7]) introduced $C$-condition as follows.

Definition 2. Let $(E, \rho)$ be a metric space and $h$ be a given self mapping on $E$. $h$ is said to satisfy C-condition if for all $t, s \in E$

$$
\frac{1}{2} \rho(t, h t) \leq \rho(t, s) \text { implies } \rho(h t, h s) \leq \rho(t, s) .
$$

Samet et al. [10] gave admissibility condition and the definition of $\alpha-\varrho$-contractive mapping as follows.

Definition 3 (see [10]). Let $(E, \rho)$ be a metric space, $h: E \longrightarrow E$ be a map, and $\alpha: E \times E \longrightarrow[0,+\infty)$ be a function.

(i) If $\alpha(t, s) \geq 1$ implies $\alpha(h t, h s) \geq 1$ for all $t, s \in E$, then $h$ is said to be $\alpha$-admissible

(ii) If $h$ is $\alpha$-admissible and $\alpha(t, z) \geq 1$ and $\alpha(t, s) \geq 1$ imply $\alpha(t, s) \geq 1$, then $h$ is said to be triangular $\alpha$-admissible.

$\Upsilon$ denotes the family of nondecreasing functions $\varrho:[0,+\infty) \longrightarrow[0,+\infty)$ such that $\sum_{n=1}^{+\infty} \varrho^{n}(t)<+\infty$ for each $t>0$, where $\varrho^{n}$ is the $n$-th iterate of $\varrho$.

Remark 1. Every function $\varrho \in \Upsilon$ satisfies $\lim _{n \longrightarrow \infty} \varrho^{n}(t)=0$ and $\varrho(t)<t$ for all $t>0$.

Definition 4 (see [10]). Let $(E, \rho)$ be a metric space and $h: E \longrightarrow E$ be a mapping. We say that $h$ is an $\alpha-\varrho-$ contractive mapping if there exist two functions $\alpha: E \times E \longrightarrow[0,+\infty)$ and $\varrho \in \Upsilon$ such that $\alpha(t, s) \rho(h t, h s) \leq \varrho(\rho(t, s))$ for all $t, s \in E$.

\section{Almost $(\alpha, \varrho)$ - Pata-Suzuki Type Contraction}

In this section, we introduce concept of almost $(\alpha, \varrho)$-Pata-Suzuki type contractions in metric spaces. We establish some fixed point results for such contractions on metric spaces.

The following lemma is necessary in our Theorem's proofs.

Lemma 1. Let $(E, \rho)$ be a metric space and $\left\{t_{n}\right\}$ be a sequence in $E$ such that $\rho\left(t_{n+1}, t_{n}\right) \longrightarrow 0$ as $n \longrightarrow \infty$. If $\left\{t_{n}\right\}$ is not a Cauchy sequence, then there exist $a \varsigma>0$ and sequences of positive integers $\left\{m_{k}\right\}$ and $\left\{n_{k}\right\}$ with $m_{k}>n_{k}>k$ such that $\rho\left(t_{m_{k}}, t_{n_{k}}\right) \geq \varsigma$ and $\rho\left(t_{m_{k}-1}, t_{n_{k}}\right) \leq \varsigma$ and $\lim _{k \longrightarrow \infty} \rho$ $\left(t_{m_{k}-1}, t_{n_{k}+1}\right)=c ̧, \quad \lim _{k \longrightarrow \infty} \rho\left(t_{m_{k}}, t_{n_{k}}\right)=\varsigma$, and $\lim _{k \longrightarrow \infty} \rho$ $\left(t_{m_{k}-1}, t_{n_{k}}\right)=\varsigma$.

From Lemma 1, we obtain $\lim _{k \longrightarrow \infty} \rho\left(t_{m_{k}+1}, t_{n_{k}+1}\right)=\varsigma$ and $\lim _{k \longrightarrow \infty} \rho\left(t_{m_{k}}, t_{n_{k}-1}\right)=\varsigma$.

Let $(E, \rho)$ be a complete metric space. Along this paper, $\Psi$ denotes the class of all increasing function $\psi:[0,1] \longrightarrow[0, \infty)$, which vanishes with continuity at zero. For an arbitrary $t_{0} \in E$, we denote $\|t\|=\rho\left(t, t_{0}\right), \forall t \in E$.

First, we begin with the following definition.

Definition 5. Let $\Lambda \geq 0, \xi \geq 1$, and $\beta \in[0, \xi]$ be fixed constants, and $\psi \in \Psi$ and $h: E \longrightarrow E$ be two functions. There exist two functions $\varrho \in \Upsilon, \alpha: E \times E \longrightarrow[0,+\infty)$ and $L \geq 0$ such that, if for all $t, s \in E$, and $\varepsilon \in[0,1], h$ satisfies the following inequality:

$$
\frac{1}{2} \rho(t, h t) \leq \rho(t, s),
$$

which implies

$$
\begin{aligned}
\alpha(t, s) \rho(h t, h s) \leq & (1-\varepsilon)(\varrho(M(t, s))+L N(t, s)) \\
& +\Lambda \varepsilon^{\xi} \psi(\varepsilon)[1+\|t\|+\|s\|+\|h t\|+\|h s\|]^{\beta},
\end{aligned}
$$

where

$$
\begin{aligned}
& M(t, s)=\max \left\{\rho(t, s), \frac{\rho(t, h t)+\rho(s, h s)}{2}, \frac{\rho(t, h s)+\rho(s, h t)}{2}\right\}, \\
& N(t, s)=\min \{\rho(t, h t), \rho(s, h s), \rho(t, h s), \rho(s, h t)\} .
\end{aligned}
$$

Then, we say that $h$ is an almost $(\alpha, \varrho)$-Pata-Suzuki contractive mapping.

Now, we are ready to prove our first result.

Theorem 2. Let $(E, \rho)$ be a complete metric space. $h: E \longrightarrow E$ be an almost $(\alpha, \varrho)-P a t a-S u z u k i$ contractive mapping. Assume that

(i) $h$ is triangular $\alpha$-admissible

(ii) There exists $x_{0} \in E$ such that $\alpha\left(t, h t_{0}\right) \geq 1$

(iii) $h$ is continuous

(iv) For all $u, v \in$ Fixh, $\alpha(u, v) \geq 1$

Then, $h$ has a unique fixed point, that is, $u=h u, u \in E$.

Proof. From the hypothesis (ii) of Theorem 2, there exists $t_{0} \in E$ such that $\alpha\left(t_{0}, h t_{0}\right) \geq 1$. Starting at the point $t_{0} \in E$, the sequence $\left\{t_{n}\right\}$ is constructed by $t_{n}=h t_{n-1}, n \geq 1$. If $t_{n_{0}+1}=$ $t_{n_{0}}$ for any $n_{0} \in \mathbb{N}$, then $x_{n_{0}}$ is a fixed point of $h$. Consequently, assume that $t_{n_{0}+1} \neq t_{n_{0}}$ for all $n_{0} \in \mathbb{N}$. First of all, we show that $\alpha\left(t_{n}, t_{n+1}\right) \geq 1$ for all $n \in \mathbb{N}$. Since $h$ is an $\alpha$-admissible mapping, we have

$$
\begin{aligned}
\alpha\left(t_{0}, t_{1}\right) \geq 1=\alpha\left(t_{0}, h t_{0}\right) & \geq 1 \text { implies } \alpha\left(t_{1}, t_{2}\right) \geq 1, \\
\alpha\left(t_{1}, t_{2}\right) & \geq 1 \text { implies } \alpha\left(t_{2}, t_{3}\right) \geq 1 .
\end{aligned}
$$

By induction, we obtain 


$$
\alpha\left(t_{n}, t_{n+1}\right) \geq 1, \quad \text { for all } n \in \mathbb{N}
$$

From the hypothesis (i) of Theorem 2, we have

$$
\begin{array}{r}
\alpha\left(t_{n}, t_{n+1}\right) \geq 1, \\
\alpha\left(t_{n+1}, t_{n+2}\right) \geq 1 \text { imply } \alpha\left(t_{n}, t_{n+2}\right) \geq 1 .
\end{array}
$$

Hence, by induction, we obtain

$$
\alpha\left(t_{n}, t_{m}\right) \geq 1, \quad \text { for all } m>n \geq 0
$$

Now, we will show that $\left\{\rho\left(t_{n+1}, t_{n}\right)\right\}$ is a decreasing sequence. Using Remark 1, since $(1 / 2) \rho\left(t_{n-1}, t_{n}\right) \leq \rho$ $\left(t_{n-1}, t_{n}\right)$, we obtain

$$
\begin{aligned}
\rho\left(t_{n+1}, t_{n}\right) \leq & (1-\varepsilon)\left(\varrho\left(\max \left\{\rho\left(t_{n}, t_{n-1}\right), \frac{\rho\left(t_{n+1}, t_{n}\right)+\rho\left(t_{n}, t_{n-1}\right)}{2}, \frac{\rho\left(t_{n}, t\right)+\rho\left(t_{n-1}, t_{n+1}\right)}{2}\right\}\right)\right. \\
& \left.+L \min \left\{\rho\left(t_{n+1}, t_{n}\right), \rho\left(t_{n}, t_{n-1}\right), \rho\left(t_{n}, t_{n}\right), \rho\left(t_{n-1}, t_{n+1}\right)\right\}\right) \\
& +\Lambda \varepsilon^{\xi} \psi(\varepsilon)\left[1+\left\|t_{n-1}\right\|+\left\|t_{n}\right\|+\left\|t_{n}\right\|+\left\|t_{n+1}\right\|\right]^{\beta} \\
\leq & (1-\varepsilon)\left(\varrho\left(\max \left\{\rho\left(t_{n}, t_{n-1}\right), \frac{\rho\left(t_{n+1}, t_{n}\right)+\rho\left(t_{n}, t_{n-1}\right)}{2}\right\}\right)\right)+K \varepsilon^{\xi} \psi(\varepsilon) \\
< & (1-\varepsilon)\left(\max \left\{\rho\left(t_{n}, t_{n-1}\right), \frac{\rho\left(t_{n+1}, t_{n}\right)+\rho\left(t_{n}, t_{n-1}\right)}{2}\right\}\right)+K \varepsilon^{\xi} \psi(\varepsilon),
\end{aligned}
$$

for some $K>0$. If $\rho\left(t_{n}, t_{n-1}\right)<\rho\left(t_{n+1}, t_{n}\right)$, then we get $\rho\left(t_{n+1}, t_{n}\right) \leq(1-\varepsilon) \rho\left(t_{n+1}, t_{n}\right)+K \varepsilon^{\xi} \psi(\varepsilon)$. In this way, we obtain $\rho\left(t_{n+1}, t_{n}\right)=0$, a contraction. Thus, we have

$$
\rho\left(t_{n+1}, t_{n}\right)<\rho\left(t_{n}, t_{n-1}\right)<\cdots<\rho\left(t_{1}, t\right)=\left\|t_{1}\right\|,
$$

that is, $\left\{\rho\left(t_{n+1}, t_{n}\right)\right\}$ is a decreasing sequence. Since $\left\{\rho\left(t_{n+1}, t_{n}\right)\right\}$ is decreasing, it is convergent to a nonnegative real number. Let $\lim _{n \longrightarrow \infty}\left(t_{n}, t_{n+1}\right)=\rho$. Now, we will show that the sequence $\left\{\left\|t_{n}\right\|\right\}$ is bounded. From triangle inequality, we have

$$
\left\|t_{n}\right\|=\rho\left(t, t_{0}\right) \leq \rho\left(t_{n}, t_{n+1}\right)+\rho\left(t_{n+1}, t_{1}\right)+\rho\left(t_{1}, t_{0}\right) .
$$

We assert a claim that

$$
\begin{gathered}
\frac{1}{2} \rho\left(t_{n}, t_{n+1}\right) \leq \rho\left(t_{n}, t_{0}\right) \\
\text { or } \frac{1}{2} \rho\left(t_{n-1}, t_{n}\right) \leq \rho\left(t_{n-1}, t_{0}\right) .
\end{gathered}
$$

On the contrary, suppose that

$$
\begin{aligned}
& \frac{1}{2} \rho\left(t_{n}, t_{n+1}\right)>\rho\left(t_{n}, t_{0}\right), \\
& \frac{1}{2} \rho\left(t_{n-1}, t_{n}\right)>\rho\left(t_{n-1}, t_{0}\right) .
\end{aligned}
$$

From triangular inequality, we have

$$
\begin{aligned}
\rho\left(t_{n-1}, t_{n}\right) \leq & \rho\left(t_{n-1}, t_{0}\right)+\rho\left(t_{0}, t_{n}\right)<\frac{1}{2}\left[\rho\left(t_{n-1}, t_{n}\right)\right. \\
& \left.+\rho\left(t_{n}, t_{n+1}\right)\right] \leq \rho\left(t_{n-1}, t_{n}\right),
\end{aligned}
$$

which is a contradiction. Thus, (14) is satisfied. Since $h$ is an almost $(\alpha, \varrho)$--Pata-Suzuki contractive mapping and using (10) and Remark 1, we obtain 


$$
\begin{aligned}
\rho\left(t_{1}, t_{n+1}\right) \leq & \alpha\left(t_{0}, t_{n}\right) \rho\left(h t_{0}, h t_{n}\right) \\
\leq & (1-\varepsilon)\left(\varrho\left(\max \left\{\rho\left(t_{n}, t_{0}\right), \frac{\rho\left(t_{n}, t_{n+1}\right)+\rho\left(t_{0}, t_{1}\right)}{2}, \frac{\rho\left(t_{n}, t_{1}\right)+\rho\left(t_{0}, t_{n+1}\right)}{2}\right\}\right)\right. \\
& \left.+L \min \left\{\rho\left(t_{n}, t_{n+1}\right), \rho\left(t_{0}, t_{1}\right), \rho\left(t_{n}, t_{1}\right), \rho\left(t_{0}, t_{n+1}\right)\right\}\right) \\
& +\Lambda \varepsilon^{\xi} \psi(\varepsilon)\left[1+\left\|t_{n}\right\|+0+\left\|t_{n+1}\right\|+\left\|t_{1}\right\|\right]^{\beta} \\
\leq & (1-\varepsilon)\left(\varrho \left(\max \left\{\rho\left(t_{n}, t_{0}\right), \frac{\rho\left(t_{n}, t_{n+1}\right)+\rho\left(t_{0}, t_{1}\right)}{2}, \frac{\rho\left(t_{n}, t_{0}\right)+\rho\left(t_{1}, t_{0}\right)+\rho\left(t_{n+1}, t_{n}\right)+\rho\left(t_{n}, t_{0}\right)}{2}\right\}\right.\right. \\
& \left.+L \min \left\{\rho\left(t_{n}, t_{n+1}\right), \rho\left(t_{0}, t_{1}\right), \rho\left(t_{n}, t_{0}\right)+\rho\left(t_{0}, t_{1}\right), \rho\left(t_{0}, t_{n+1}\right)\right\}\right) \\
& +\Lambda \varepsilon^{\xi} \psi(\varepsilon)\left[1+2\left\|t_{n}\right\|+2\left\|t_{1}\right\|\right]^{\beta} \\
\leq & (1-\varepsilon)\left(\varrho\left(\max \left\{\left\|t_{n}\right\|,\left\|t_{1}\right\|,\left\|t_{n}\right\|+\left\|t_{1}\right\|\right\}\right)+L \min \left\{\left\|t_{1}\right\|,\left\|t_{n}\right\|+\left\|t_{1}\right\|,\left\|t_{n+1}\right\|\right\}\right)+\Lambda \varepsilon^{\xi} \psi(\varepsilon)\left[1+2\left\|t_{n}\right\|+2\left\|t_{1}\right\|\right]^{\beta} \\
\leq & (1-\varepsilon)\left(\varrho\left(\left\|t_{n}\right\|+\left\|t_{1}\right\|\right)+L\left\|t_{n+1}\right\|\right)+\Lambda \varepsilon^{\xi} \psi(\varepsilon)\left[1+2\left\|t_{n}\right\|+2\left\|t_{1}\right\|\right]^{\beta} \\
< & \left(\left\|t_{n}\right\|+\left\|t_{1}\right\|+L\left\|t_{n+1}\right\|\right)+\Lambda \varepsilon^{\xi} \psi(\varepsilon)\left[1+2\left\|t_{n}\right\|+2\left\|t_{1}\right\|\right]^{\beta} .
\end{aligned}
$$

Using $\beta \leq \xi$, we get

$$
\begin{aligned}
& \left\|t_{n}\right\|<(1-\varepsilon)\left(\left\|t_{n}\right\|+\left\|t_{1}\right\|+L\left\|t_{1}\right\|\right)+2\left\|t_{1}\right\|+\Lambda \varepsilon^{\xi} \psi(\varepsilon)\left[1+2\left\|t_{n}\right\|+2\left\|t_{n+1}\right\|\right]^{\xi}, \\
& \varepsilon\left\|t_{n}\right\|<k \varepsilon^{\xi} \psi(\varepsilon)\left\|t_{n}\right\|^{\xi}+l
\end{aligned}
$$

for some $k, l>0$. By the same reason as in [13], the sequence $\left\|\left\{t_{n}\right\}\right\|$ is bounded. Let $\lim _{n \longrightarrow \infty} \rho\left(t_{n}, t_{n+1}\right)=\rho$. Using Remark 1 and from (8), we have

$$
\begin{aligned}
\rho\left(t_{n}, t_{n+1}\right) \leq & \alpha\left(t_{n-1}, t_{n}\right) \rho\left(t_{n}, t_{n+1}\right) \\
\leq & (1-\varepsilon)\left(\left(\varrho\left(\max \left\{\rho\left(t_{n}, t_{n-1}\right), \frac{\rho\left(t_{n+1}, t\right)+\rho\left(t_{n}, t_{n-1}\right)}{2}, \frac{\rho\left(t_{n}, t_{n}\right)+\rho\left(t_{n-1}, t_{n+1}\right)}{2}\right\}\right)\right)\right. \\
& \left.+L \min \left\{\rho\left(t_{n+1}, t_{n}\right), \rho\left(t_{n}, t_{n-1}\right), \rho\left(t_{n}, t_{n}\right), \rho\left(t_{n-1}, t_{n+1}\right)\right\}\right) \\
& +\Lambda \varepsilon^{\xi} \psi(\varepsilon)\left[1+\left\|t_{n}\right\|+\left\|t_{n-1}\right\|+\left\|t_{n+1}\right\|+\left\|t_{n}\right\|\right]^{\beta} \\
\leq & (1-\varepsilon) \varrho\left(\max \left\{\rho\left(t_{n}, t_{n-1}\right), \frac{\rho\left(t_{n+1}, t_{n}\right)+\rho\left(t_{n}, t_{n-1}\right)}{2}\right\}\right)+K \varepsilon^{\xi} \psi(\varepsilon), \\
< & (1-\varepsilon) \max \left\{\rho\left(t_{n}, t_{n-1}\right), \frac{\rho\left(t_{n+1}, t_{n}\right)+\rho\left(t_{n}, t_{n-1}\right)}{2}\right\}+K \varepsilon^{\xi} \psi(\varepsilon),
\end{aligned}
$$


for some $K>0$. Taking limit as $n \longrightarrow \infty$, we obtain $\rho \leq K \varepsilon^{\xi} \psi(\varepsilon)$ and thus $\rho=0$.

Next, we demonstrate that $\left\{t_{n}\right\}$ is a Cauchy sequence. Assume that the sequence $\left\{t_{n}\right\}$ is not a Cauchy sequence. From Lemma 1, there exist subsequence $\left\{t_{m_{k}}\right\}$ and $\left\{t_{n_{k}}\right\}$ with $n_{k}>m_{k}>k$ such that $\quad \lim _{k \longrightarrow \infty} \rho\left(t_{m_{k}-1}, t_{n_{k}+1}\right)=\varsigma$, $\lim _{k \longrightarrow \infty} \rho\left(t_{m_{k}}, t_{n_{k}}\right)=\varsigma, \quad \quad \lim _{k \longrightarrow \infty} \rho\left(t_{m_{k}-1}, t_{n_{k}}\right)=\varsigma$, $\lim _{k \longrightarrow \infty} \rho\left(t_{m_{k}+1}, t_{n_{k}+1}\right)=\varsigma$, and $\lim _{k \longrightarrow \infty} \rho\left(t_{m_{k}}, t_{n_{k}-1}\right)=\varsigma$. We assert that

$$
\frac{1}{2} \rho\left(t_{m_{k}-1}, t_{m_{k}}\right) \leq \rho\left(t_{m_{k}-1}, t_{n_{k}-1}\right) .
$$

If we assume that

$$
\frac{1}{2} \rho\left(t_{m_{k}-1}, t_{m_{k}}\right)>\rho\left(t_{m_{k}-1}, t_{n_{k}-1}\right),
$$

then we obtain a contradiction. If we take limit $k \longrightarrow \infty$ in inequality (21), we obtain contradiction. Since $h$ is an almost $(\alpha, \varrho)$-Pata-Suzuki contractive mapping, we have

$$
\begin{aligned}
\varsigma \leq & \rho\left(t_{m_{k}}, t_{n_{k}}\right)=\rho\left(h t_{m_{k}-1}, h t_{n_{k}-1}\right) \\
\leq & \alpha\left(t_{m_{k}}, t_{n_{k}}\right) \rho\left(h t_{m_{k}-1}, h t_{n_{k}-1}\right) \\
\leq & (1-\varepsilon)\left(\varrho\left(\max \left\{\rho\left(t_{m_{k}-1}, t_{n_{k}-1}\right), \frac{\rho\left(t_{m_{k}-1}, t_{m_{k}}\right)+\rho\left(t_{n_{k}-1}, t_{n_{k}}\right)}{2}, \frac{\rho\left(t_{n_{k-1}}, t_{m_{k}}\right)+\rho\left(t_{m_{k}-1}, t_{n_{k}}\right)}{2}\right\}\right)\right. \\
& \left.+L \min \left\{\rho\left(t_{m_{k}-1}, t_{m_{k}}\right), \rho\left(t_{n_{k}-1}, t_{n_{k}}\right), \rho\left(t_{n_{k-1}}, t_{m_{k}}\right), \rho\left(t_{m_{k}-1}, t_{n_{k}}\right)\right\}\right) \\
& +\Lambda \varepsilon^{\xi} \psi(\varepsilon)\left[1+\left\|t_{m_{k}}\right\|+\left\|t_{n_{k}}\right\|+\left\|t_{n_{k}+1}\right\|+\left\|t_{m_{k}+1}\right\|\right]^{\beta} \\
< & (1-\varepsilon)\left(\max \left\{\rho\left(t_{m_{k}-1}, t_{n_{k}-1}\right), \frac{\rho\left(t_{m_{k}-1}, t_{m_{k}}\right)+\rho\left(t_{n_{k}-1}, t_{n_{k}}\right)}{2}, \frac{\rho\left(t_{n_{k-1}}, t_{m_{k}}\right)+\rho\left(t_{m_{k}-1}, t_{n_{k}}\right)}{2}\right\}\right. \\
& \left.+L \min \left\{\rho\left(t_{m_{k}-1}, t_{m_{k}}\right), \rho\left(t_{n_{k}-1}, t_{n_{k}}\right), \rho\left(t_{n_{k-1}}, t_{m_{k}}\right), \rho\left(t_{m_{k}-1}, t_{n_{k}}\right)\right\}\right) \\
& +\Lambda \varepsilon^{\xi} \psi(\varepsilon)\left[1+\left\|t_{m_{k}}\right\|+\left\|t_{n_{k}}\right\|+\left\|t_{n_{k}+1}\right\|+\left\|t_{m_{k}+1}\right\|\right]^{\beta} .
\end{aligned}
$$

Taking the limit as $k \longrightarrow \infty$, we obtain

$$
\varsigma \leq(1-\varepsilon) \varsigma+K \varepsilon \psi(\varepsilon)
$$

and then

$$
\varsigma \leq K \psi(\varepsilon),
$$

that is, $\varsigma=0$, which is a contradiction. Hence, the sequence $\left\{t_{n}\right\}$ is a Cauchy sequence in $(E, \rho)$. By the completeness of $E$, the sequence $\left\{t_{n}\right\}$ is convergent to some $u \in E$, that is, $t_{n} \longrightarrow u$ as $n \longrightarrow+\infty$. Since $h$ is continuous, $h t_{n} \longrightarrow h u$ as $n \longrightarrow+\infty$. By the uniqueness of the limit, we obtain $u=h u$; that is, $u$ is a fixed point of $h$.

Now, we observe that fixed point of $h$ is unique. Assume that $u$ and $v$ are fixed points of $h$. Since $h$ satisfies the hypothesis (iv) of Theorem 2, h is an almost $(\alpha, \varphi)$-Pata-Suzuki contractive mapping, Remark 1, and $(1 / 2) \rho(u, h u) \leq \rho(u, v)$, we have

$\rho(h u, h v) \leq \alpha(u, v) \rho(h u, h v)$

$$
\begin{aligned}
\leq & (1-\varepsilon)\left(\varrho\left(\max \left\{\rho(u, v), \frac{\rho(u, h u)+\rho(v, h v)}{2}, \frac{\rho(u, h v)+\rho(v, h u)}{2}\right\}\right)\right. \\
& +L \min \{\rho(u, h u), \rho(v, h v), \rho(u, h v), \rho(v, h u)\})+K \varepsilon \psi(\varepsilon) \\
< & (1-\varepsilon)\left(\max \left\{\rho(u, v), \frac{\rho(u, h u)+\rho(v, h v)}{2}, \frac{\rho(u, h v)+\rho(v, h u)}{2}\right\}\right. \\
& +L \min \{\rho(u, h u), \rho(v, h v), \rho(u, h v), \rho(v, h u)\})+K \varepsilon \psi(\varepsilon) .
\end{aligned}
$$


Hence, we obtain that $\rho(u, v) \leq K \psi(\varepsilon)$, and thus $u=v$. Hence, $h$ has a unique fixed point in $E$.

Now, we prove the following theorem without the assumption of continuity of $h$.

Theorem 3. Let $(E, \rho)$ be a complete metric space. $h: E \longrightarrow E$ be an almost $(\alpha, \varphi)-$ Pata-Suzuki contractive mapping. Assume that

(i) $h$ is triangular $\alpha$-admissible

(ii) There exists $t_{0} \in E$ such that $\alpha\left(t_{0}, h t_{0}\right) \geq 1$

(iii) If $\left\{t_{n}\right\}$ is a sequence in E such that $\alpha\left(t_{n}, t_{n+1}\right) \geq 1$ for all $n$ and $t_{n} \longrightarrow u \in E$ as $n \longrightarrow+\infty$, then $\alpha\left(t_{n}, u\right) \geq 1$ for all $n$

(iv) For all $u, v \in$ Fixh, $\alpha(u, v) \geq 1$

Then, $h$ has a unique fixed point, that is, $u=h u, u \in E$.
Proof. Following the proof of Theorem 2, we have already known that $\left\{t_{n}\right\}$ is a Cauchy sequence in $E$. Since $E$ is complete, we have $t_{n} \longrightarrow u \in E$ as $n \longrightarrow+\infty$. Now, we prove that $u=h u$. From (8) and hypothesis (iii) of Theorem 3 , we have $\alpha\left(t_{n}, u\right) \geq 1$ for all $n$. For all $n \geq 1$, we assert that

$$
\begin{aligned}
\frac{1}{2} \rho\left(t_{n}, t_{n+1}\right) & \leq \rho\left(t_{n}, u\right) \\
\text { or } \frac{1}{2} \rho\left(t_{n-1}, t_{n}\right) & \leq \rho\left(t_{n-1}, u\right) .
\end{aligned}
$$

We assume that

$$
\begin{aligned}
& \frac{1}{2} \rho\left(t_{n}, t_{n+1}\right)>\rho\left(t_{n}, u\right), \\
& \frac{1}{2} \rho\left(t_{n-1}, t_{n}\right)>\rho\left(t_{n-1}, u\right) .
\end{aligned}
$$

Since $\left\{\rho\left(t_{n+1}, t_{n}\right)\right\}$ is a decreasing sequence,

$$
\rho\left(t_{n-1}, t_{n}\right) \leq \rho\left(t_{n-1}, u\right)+\rho\left(u, t_{n}\right)<\frac{1}{2}\left[\rho\left(t_{n-1}, t_{n}\right)+\rho\left(t_{n}, t_{n+1}\right)\right] \leq \rho\left(t_{n-1}, t_{n}\right)
$$

which is a contradiction. Thus, (26) is held, and we have

$$
\begin{aligned}
\rho(h u, u)= & \rho\left(h u, t_{n+1}\right)+\rho\left(t_{n+1}, u\right) \\
\leq & \alpha\left(t_{n}, u\right) \rho\left(h u, t_{n+1}\right)+\rho\left(t_{n+1}, u\right) \\
\leq & (1-\varepsilon)\left(\varrho\left(\max \left\{\rho\left(u, t_{n}\right), \frac{\rho(u, h u)+\rho\left(t_{n}, t_{n+1}\right)}{2}, \frac{\rho\left(u, t_{n+1}\right)+\rho\left(t_{n}, h u\right)}{2}\right\}\right)\right. \\
& \left.+L \min \left\{\rho(u, h u), \rho\left(t_{n}, t_{n+1}\right), \rho\left(u, t_{n+1}\right), \rho\left(t_{n}, h u\right)\right\}\right) \\
& +\Lambda \varepsilon^{\xi} \psi(\varepsilon)\left[1+\left\|t_{n}\right\|+\|u\|+\|h u\|+\left\|t_{n+1}\right\|\right]^{\beta}+d\left(t_{n+1}, u\right) \\
< & \max \left\{\rho\left(u, t_{n}\right), \frac{\rho(u, t u)+\rho\left(t_{n}, t_{n+1}\right)}{2}, \frac{\rho\left(u, t_{n+1}\right)+\rho\left(t_{n}, h u\right)}{2}\right\} \\
& +L \min \left\{\rho(u, h u), \rho\left(t_{n}, t_{n+1}\right), \rho\left(u, t_{n+1}\right), \rho\left(t_{n}, h u\right)\right\}+K \varepsilon^{\xi} \psi(\varepsilon)+\rho\left(t_{n+1}, u\right),
\end{aligned}
$$

for some $K>0$. Taking the limit as $n \longrightarrow \infty$, we obtain

$$
\rho(h u, u) \leq \frac{1}{2} \rho(h u, u)+K \varepsilon^{\xi} \psi(\varepsilon)
$$

Thus, we make an inference that $h u=u$ and that is $u$ is a fixed point of $h$. Similar to the proof of Theorem 2 the uniqueness of fixed point of $h$ can be obtained.

We give an example related to our main theorem.

Example 1. Let $E=[0,1]$ with the usual metric and define the mapping $h: E \longrightarrow E$ by $h(t)=(t / 3)$. Let $\varrho:[0,+\infty) \longrightarrow 0,+\infty)$ be defined as $\varrho(t)=(t / 2)$ and $\alpha: E \times E \longrightarrow[0,+\infty), \alpha(t, s)=\left\{\begin{array}{ll}1 & t, s \in[0,1] \\ 0 & t, s \notin[0,1]\end{array}\right.$. It is easy to get $h$ is triangular $\alpha$-admissible. Our aim is to prove that $h$ satisfies (5). For $x, y \in[0,1]$,

$$
\frac{1}{2} \rho(t, h t)=\frac{1}{2}\left|t-\frac{t}{3}\right| \leq \rho(t, s)
$$

which implies

$$
\rho(h t, h s)=\alpha(t, s) \rho(h t, h s)=\left|\frac{t}{3}-\frac{s}{3}\right|=\frac{1}{3} \rho(t, s) .
$$

Since $\rho(t, s) \leq M(t, s)$, we obtain 


$$
\rho(h t, h s) \leq \frac{1}{3} M(t, s)=\frac{2}{3} \varrho(M(t, s)),
$$

For arbitrary $\varepsilon \in[0,1]$, we can write inequality (34) as and for $L=0$, we can write

$$
\rho(h t, h s) \leq \frac{2}{3} \varrho(M(t, s))+L N(t, s) .
$$
follows:

$$
\begin{aligned}
\rho(h t, h s) & \leq(1-\varepsilon)(\varrho(M(t, s))+L N(t, s))+\left(\frac{2}{3}+\varepsilon-1\right) M(t, s) \\
& \leq(1-\varepsilon)(\varrho(M(t, s))+L N(t, s))+\left(\frac{2}{3}+\varepsilon-1\right)(1+\|t\|+\|s\|+\|h t\|+\|h s\|) .
\end{aligned}
$$

Our aim is to prove that $\gamma \geq 0$ and $\Lambda \geq 0$ such that

$$
\left(\frac{2}{3}+\varepsilon-1\right)(1+\|t\|+\|s\|+\|h t\|+\|h s\|) \leq \Lambda \varepsilon^{\gamma+1}(1+\|t\|+\|s\|+\|h t\|+\|h s\|)
$$

which satisfies for all $t, s \in[0,1]$, and every $0 \leq \varepsilon \leq 1$. We can find $\Lambda \geq 0$ such that

$$
\Lambda=\frac{(2 / 3)+\varepsilon-1}{\varepsilon^{\gamma+1}}
$$

which satisfies for each $0 \leq \varepsilon \leq 1$ and some $\gamma \geq 0$. If we choose $\gamma$ such that $(\gamma / \gamma+1)>1-(2 / 3)$, then

$$
\Lambda=\frac{\gamma^{\gamma}}{(\gamma+1)^{\gamma+1}(1-(2 / 3))^{\gamma}} .
$$

Thus, we have that

$$
\rho(t, h t)=\left|t-\frac{t}{3}\right| \leq \rho(t, s),
$$

which implies

$$
\alpha(t, s) \rho(h t, h s) \leq(1-\varepsilon)(\varrho(M(t, s))+L N(t, s))+\Lambda \varepsilon^{\gamma+1}(1+\|t\|+\|s\|+\|h t\|+\|h s\|)
$$

which satisfies for all $t, s \in[0,1]$ and each $\varepsilon>0$. If $\varepsilon=0$, it can be seen that (5) is satisfied. Hence, the conditions of Theorem 2 are satisfied with $\psi(\varepsilon)=\varepsilon^{\gamma}, \xi=\beta=1$. By an application of Theorem $2, h$ has a unique fixed point in $E=[0,1]$. It is seen that $u=0$ is the unique fixed point of $h$ in $E$.

The following result is directly obtained from Theorem 2 by taking $\varepsilon=0$.

Corollary 1. Let $h: E \longrightarrow E$ be a function. There exist two functions $\varphi \in Y$ and $\alpha: E \times E \longrightarrow[0,+\infty)$ and $L \geq 0$ such that if for all $t, s \in E, h$ satisfies the inequality

$$
\frac{1}{2} \rho(t, h t) \leq \rho(t, s),
$$

which implies

$$
\alpha(t, s) \rho(h t, h s) \leq \varrho(M(t, s))+L N(t, s),
$$

where

$$
\begin{aligned}
& M(t, s)=\max \left\{\rho(t, s), \frac{\rho(t, h t)+\rho(s, h s)}{2}, \frac{\rho(t, h s)+\rho(s, h t)}{2}\right\}, \\
& N(t, s)=\min \{\rho(t, h t), \rho(s, h s), \rho(t, h t), \rho(s, h t)\},
\end{aligned}
$$

and assume that

(i) $h$ is triangular $\alpha$-admissible

(ii) There exists $t_{0} \in E$ such that $\alpha\left(t_{0}, h t_{0}\right) \geq 1$

(iii) $h$ is continuous

(iv) For all $u, v \in$ Fixh, $\alpha(u, v) \geq 1$

Then, $h$ has a unique fixed point, that is, $u=h u, u \in E$. 
Corollary 1 generalizes the results of Samet [10] and Karapinar [11].

Corollary 2. Let $\Lambda \geq 0, \xi \geq 1$ and $\beta \in[0, \xi]$ be fixed constants and $h: E \longrightarrow E$ be a function. There exist two functions $\psi \in \Psi$ and $\alpha: E \times E \longrightarrow[0,+\infty)$ and $L \geq 0$ such that if for all $t, s \in E$, and $\varepsilon \in[0,1]$, $h$ satisfies the inequality

$$
\frac{1}{2} \rho(t, h t) \leq \rho(t, s)
$$

which implies

$$
\alpha(t, s) \rho(h t, h s) \leq(1-\varepsilon)(M(t, s)+L N(t, s))+\Lambda \varepsilon^{\xi} \psi(\varepsilon)[1+\|t\|+\|s\|+\|h t\|+\|h s\|]^{\beta},
$$

where

$$
\begin{aligned}
& M(t, s)=\max \left\{\rho(t, s), \frac{\rho(t, h t)+\rho(s, h s)}{2}, \frac{\rho(t, h s)+\rho(s, h t)}{2}\right\}, \\
& N(t, s)=\min \{\rho(t, h t), \rho(s, h s), \rho(t, h s), \rho(s, h y)\}
\end{aligned}
$$

and assume that

(i) $h$ is triangular $\alpha$-admissible

(ii) There exists $t_{0} \in E$ such that $\alpha\left(t_{0}, h t_{0}\right) \geq 1$

(iii) $h$ is continuous

(iv) For all $u, v \in$ Fixh, $\alpha(u, v) \geq 1$
Then, $h$ has a unique fixed point that is $u=h u, u \in E$.

If we take $L=0$ in Theorem 2 , then we say that $h$ is $(\alpha, \varrho)-$ Pata-Suzuki contractive mapping and get the following corollary.

Corollary 3. Let $\Lambda \geq 0, \xi \geq 1$, and $\beta \in[0, \xi]$ be fixed constants; $\psi \in \Psi$ and $h: E \longrightarrow E$ be two functions. There exist two functions $\varrho \in Y$ and $\alpha: E \times E \longrightarrow[0,+\infty)$ such that if for all $t, s \in E$, and $\varepsilon \in[0,1]$, $h$ satisfies the inequality

$$
\frac{1}{2} \rho(t, h t) \leq \rho(t, s),
$$

which implies

$$
\alpha(t, s) \rho(h t, h s) \leq(1-\varepsilon) \varrho(M(t, s))+\Lambda \varepsilon^{\xi} \psi(\varepsilon)[1+\|t\|+\|s\|+\|h t\|+\|h s\|]^{\beta}
$$

where

$$
M(t, s)=\max \left\{\rho(t, s), \frac{\rho(t, h t)+\rho(s, h s)}{2}, \frac{\rho(t, h s)+\rho(s, h t)}{2}\right\},
$$

and assume that

(i) $h$ is triangular $\alpha$-admissible

(ii) There exists $t_{0} \in E$ such that $\alpha\left(t_{0}, h t_{0}\right) \geq 1$

(iii) $h$ is continuous

(iv) For all $u, v \in$ Fixh, $\alpha(u, v) \geq 1$

Then, $h$ has a unique fixed point, that is, $u=h u, u \in E$.
If we take $\alpha(t, s)=1$ for all $t, s \in E, M(t, s)=\rho(t, s)$ and $N(t, s)=\rho(s, h t)$, in Theorem 2 , then we get the following corollary.

Corollary 4. Let $(E, \rho)$ be a complete metric space, $\Lambda \geq 0$, $\xi \geq 1$, and $\beta \in[0, \xi]$ be fixed constants, and $\psi \in \Psi, \varrho \in Y$, and $h: E \longrightarrow E$ be a function. There exist a function $\varphi \in \Upsilon$ and $L \geq 0$ such that if for all $t, s \in E$, $h$ satisfies the inequality

$$
\frac{1}{2} \rho(t, h t) \leq \rho(t, s),
$$

which implies

$$
\rho(h t, h s) \leq(1-\varepsilon)(\varrho(\rho(t, s))+L \rho(s, h t))+\Lambda \varepsilon^{\xi} \psi(\varepsilon)[1+\|t\|+\|s\|+\|h t\|+\|h s\|]^{\beta},
$$

is satisfied for all $\varepsilon \in[0,1]$, and then $h$ has a unique fixed point $u=h u, u \in E$.

If we take $\alpha(t, s)=1$ for all $t, s \in E, M(t, s)=\rho(t, s)$ and $N(t, s)=\rho(s, h t), \varrho(t)<t$, for all $t>0$, in Theorem 2 , then we get the following corollary.
Corollary 5. Let $(E, \rho)$ be a complete metric space, $\Lambda \geq 0$, $\xi \geq 1$, and $\beta \in[0, \xi]$ be fixed constants, and $\psi \in \Psi$ and $h: E \longrightarrow E$ be a function. There exists $L \geq 0$ such that if for all $t, s \in E$, and $\varepsilon \in[0,1]$, $h$ satisfies the inequality

$$
\frac{1}{2} \rho(t, h t) \leq \rho(t, s),
$$


which implies

$$
\rho(h t, h s) \leq(1-\varepsilon)(\rho(t, s)+L \rho(s, h t))+\Lambda \varepsilon^{\xi} \psi(\varepsilon)[1+\|t\|+\|s\|+\|h t\|+\|h s\|]^{\beta},
$$

is satisfied for all $\varepsilon \in[0,1]$, and then $h$ has a unique fixed point $u=h u, u \in E$.
Corollary 6. Let $(E, \rho)$ be a complete metric space, $\Lambda \geq 0$, $\xi \geq 1$, and $\beta \in[0, \xi]$ be fixed constants, and $\psi \in \Psi$ and $h: E \longrightarrow E$ be a function. There exists $L \geq 0$ such that if for all $t, s \in E$, and $\varepsilon \in[0,1]$, $h$ satisfies the inequality

$$
\rho(h t, h s) \leq(1-\varepsilon)(\rho(t, s)+L \rho(s, h t))+\Lambda \varepsilon^{\xi} \psi(\varepsilon)[1+\|t\|+\|s\|+\|h t\|+\|h s\|]^{\beta},
$$

which is satisfied for all $\varepsilon \in[0,1]$, and then $h$ has a unique fixed point $u=h u, u \in E$.

Corollary 6 generalizes the results of Pata [13] and Banach [1].

\section{Data Availability}

The data used to support the findings of this study are included in the references within the article.

\section{Conflicts of Interest}

The authors declare that they have no conflicts of interest.

\section{Acknowledgments}

The first author would like to thank TUBITAK (the Scientific and Technological Research Council of Turkey) for their financial supports during her $\mathrm{PhD}$ studies.

\section{References}

[1] S. Banach, "Sur les opérationes dans les ensembles abstraits et leur application aux équation intégrales," Fundamenta Mathematicae, vol. 3, pp. 133-181, 1922.

[2] V. Berinde and M. Pacurar, "Fixed points and continuity of almost contractions," Fixed Point Theory, vol. 9, pp. 23-34, 2008.

[3] V. Berinde, "Approximating fixed points of weak contraction using the picard iteration," Nonlinear Analysis Forum, vol. 9, pp. 43-53, 2004.

[4] M. Berinde and V. Berinde, "On general class of multivalued weakly picard mappings," Journal of Mathematical Analysis and Applications, vol. 326, no. 2, pp. 772-782, 2007.

[5] T. Suzuki, "A new type of fixed point theorem in metric spaces," Nonlinear Analysis: Theory, Methods \& Applications, vol. 71, no. 11, pp. 5313-5317, 2009.

[6] T. Suzuki, "Some results on recent generalization of Banach contraction principle," in Proceedings of the 8th International Conference of Fixed Point Theory and its Applications, pp. 751-761, Chiang Mai, Thailand, July 2007.

[7] T. Suzuki, "Fixed point theorems and convergence theorems for some generalized nonexpansive mappings," Journal of
Mathematical Analysis and Applications, vol. 340, no. 2, pp. 1088-1095, 2008.

[8] T. Suzuki, "A generalized banach contraction principle which characterizes metric completeness," Proceedings of the American Mathematical Society, vol. 136, pp. 1861-1869, 2008.

[9] M. Edelstein, "On fixed and periodic points under contractive mappings," Journal of the London Mathematical Society, vol. s1-37, no. 1, pp. 74-79, 1962.

[10] B. Samet, C. Vetro, and P. Vetro, "Fixed point theorem for $(\alpha, \psi)$-contractive type mapping," Nonlinear Analysis: Theory, Methods \& Applications, vol. 75, no. 4, pp. 2154-2165, 2012.

[11] E. Karapinar and B. Samet, "Generalized $(\alpha, \psi)$-contractive type mappings and related fixed point theorems with applications," Abstract and Applied Analysis, vol. 2012, 17 pages, Article ID 793486, 2012.

[12] G. V. R. Babu, K. K. M. Sarma, and V. A. Kumari, "Fixed points of almost generalized $(\alpha, \psi)$-contractions with rational expressions," Mathematical Journal Of Interdisciplinary Sciences, vol. 5, no. 2, pp. 101-120, 2017.

[13] V. Pata, "A fixed point theorem in metric spaces," Journal of Fixed Point Theory and Applications, vol. 10, no. 2, pp. 299305, 2011.

[14] S. Balasubramanian, "A pata-type fixed point theorem," Mathematical Sciences, vol. 8, no. 3, pp. 65-69, 2014.

[15] Z. Kadelburg and S. Radenović, "Fixed point theorems under pata-type conditions in metric spaces," Journal of the Egyptian Mathematical Society, vol. 24, no. 1, pp. 77-82, 2016.

[16] M. Paknazar, M. Eshaghi, Y. J. Cho et al., "A pata-type fixed point theorem in modular spaces with application," Fixed Point Theory Applications, vol. 239, pp. 1687-1812, 2013.

[17] M. Chakraborty and S. K. Samanta, "On a fixed point theorem for a cyclical kannan-type mapping," Facta Universitatis Series Mathematics Informatics, vol. 28, pp. 179-188, 2013.

[18] M. Eshaghi, S. Mohseni, M. R. Delavar, M. De La Sen, G. Kim, and A. Arian, "Pata contractions and coupled type fixed points," Fixed Point Theory and Applications, vol. 2014, no. 1, p. 130, 2014. 